\title{
Detection of Vascular Morphology by High Frequency Intravascular Ultrasonic Imaging
}

\author{
WILHELMINA J. GUSSENHOVEN, STANLEY MADRETSMA, HERMAN PIETERMAN, \\ SALEM H.K. THE, LI WENGUANG, FRANS VAN EGMOND, NICOLAAS BOM \\ Thoraxcenter, University Hospital Dijkzigt, Erasmus University Rotterdam, and the Interuniversity Cardiology Institute of The \\ Netherlands \\ Correspondence to W.J. Gussenhoven MD, Erasmus University (Ee 2312), P.O Box 1738, 3000 DR Rotterdam, The \\ Netherlands
}

\begin{abstract}
This study was designed to validate the potential clinical utility of intravascular ultrasonic imaging in vitro and in vivo. In vitro studies were performed to assess the accuracy of dimensional and morphological information. In vitro images of human vessels $(n=75)$ demonstrated that lesion thickness determined echographically closely related with histological samples $(r=0.83)$. Morphologically, muscular and elastic arteries could be distinguished echographically based on the echogenicity of the arterial media. Close relation was also found in the morphological subtypes of atherosclerosis.

Subsequently, intravascular ultrasound was used percutaneously in vivo in 20 patients to obtain images of the iliac and superficial femoral artery. High quality real-time images were obtained. Normal vessels were seen showing pulsatile circular images with a hypoechoic muscular media resulting in a typical three-layered appearance. Diseased arteries revealed non-obstructive and obstructive lumen. At the site of obstruction thinning of the muscular media was evident. Pulsation was not always present. Following dilatation of the obstructive lesion using balloon angioplasty the ultrasonic cross-sections changed drastically revealing plaque rupture, dissection, plaque-free wall rupture, rest stenosis and oedema.

We conclude that intravascular ultrasonic imaging is a promising technique to document accurate dimensional and morphological characteristics of human vascular disease for guidance of therapeutic interventions.
\end{abstract}

\section{INTRODUCTION}

The necessity for more detailed morphological information on the arterial anatomy has accelerated the current advances in the development of high frequency intravascular ultrasound systems. A successful application of such a system may contribute important prognostic information, especially with regard to the interventional therapy for peripheral and coronary artery disease. Recent intravascular ultrasound systems are either based on a mechanically rotated acoustic element or electronically switched elements. Mechanically driven catheter tip echo systems operate with a flexible shaft. There are two types: the rotary element; and the rotary mirror technique (1). These systems provide a real-time crosssectional observation of the artery (1-7).
In vitro and in vivo studies from our laboratory have shown that high frequency intravascular ultrasound imaging based on the mechanical rotating principle provides information on lumen size, the vascular anatomy, morphology and location of the atherosclerotic lesion (8-11).

After a brief description of the intravascular echo system as currently available at our centre, the potential of this technique for qualification and quantification purposes will be discussed.

\section{MATERIALS AND METHODS}

Details of the intravascular ultrasound device used have been described earlier (4). Briefly, it is a mechanically driven catheter tip system 


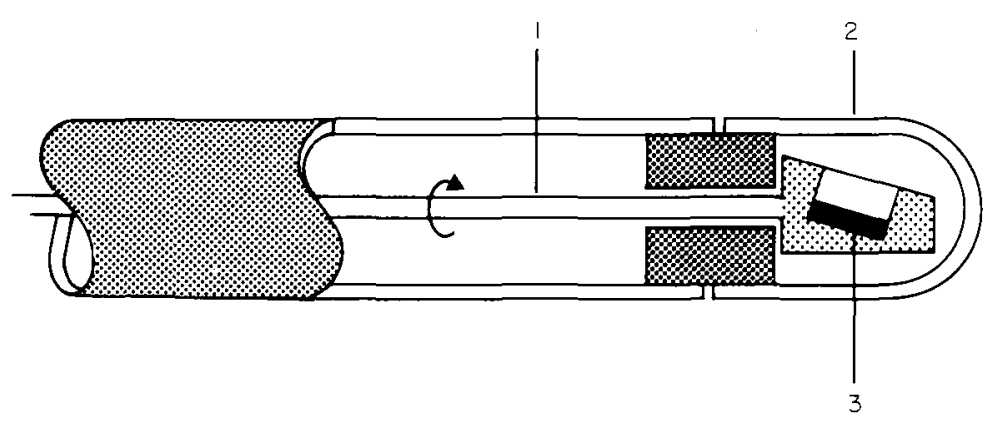

Fig. 1. Rotating tip system is schematically illustrated. The shaft (1) is flexible and contains the electric wires for the transducer. The element (2) is positioned in a $15^{\circ}$ angle so that no transmission pulse effect caused by the dome appears on the display (3).

using a flexible shaft (Fig. 1). Within the shaft are the electric wires for the $30 \mathrm{MHz}$ transducer, which can be rotated up to 1000 r.p.m. The single element transducer is positioned such that no transmission pulse effect appears on the display, as the echo travel time to the acoustically transparent dome is of sufficient duration to prevent this. Imaging very close to the catheter outer wall is possible and no 'dead zone' is present. The $5 \mathrm{~F}$ catheter has a length of $90 \mathrm{~cm}$. The ultrasound beam is emitted at a $15^{\circ}$ look-forward angle. Axial resolution is $80 \mu \mathrm{m}$ and lateral resolution $225 \mu \mathrm{m}$ at a depth of $1 \mathrm{~mm}$. All images were stored on a videosystem.

For in vitro studies we used human arterial specimens (stored frozen at $-20^{\circ} \mathrm{C}$ ). For in vitro studies the segments were thawed and embedded vertically in a $1.2 \%$ solution of agar-agar. Experiments in vitro were carried out by placing the catheter inside the vascular lumen which was filled with saline. The crosssections were obtained of the specimen from proximal to distal by positioning the transducer slightly more distally into the specimens. The morphology of the vessel interrogated as well as the location, extension, thickness and morphology of the atherosclerotic lesion were documented and compared with the matched histologic cross-section (vide infra).

In vivo, serial cross-sections were obtained from 20 patients. Peripheral vessels including aorta, iliac, femoral and popliteal arteries were examined.

\section{Microscopic examination}

Following ultrasound examination, the proximal site of the arteries were marked with ink corresponding to the echographic 12 o'clock position. The arteries were fixed in $10 \%$ buf- fered formalin for $12 \mathrm{~h}$ and subsequently decalcified in a standard Cal Ex solution (Fisher Inc., USA) for $5 \mathrm{~h}$. The arteries were then processed for routine paraffin embedding. Transverse sections of $5 \mu \mathrm{m}$ thick were cut with $1 \mathrm{~mm}$ intervals between each, ensuring that the transverse sections were perpendicular to the longitudinal axis of the artery. The histologic sections were stained by the haematoxylinazophloxine technique and with the Verhoeff's elastin van Gieson technique. With the latter technique a highly selective black staining of elastin fibres is achieved. The van Gieson technique is used as counterstaining for muscle and connective tissue (12). Under lightmicroscopy the nature of the atherosclerotic lesion as well as the maximal plaque thickness was established. Histological cross-sections were paired with the corresponding echo image. For comparison purposes one cross-section of each artery showing the most significant plaque thickness was selected. Photographs were made of these sections together with a calibrator indicating a $1 \mathrm{~mm}$ scale in depth. The cross-sections were compared for morphological purposes and for quantification.

\section{Quantification}

Maximal plaque thickness was determined by two observers as the distance between the surface of the plaque up to the internal elastic lamina junction to the media. Boundaries of the plaque surface with the lumen were always distinct, both on histologic and echographic examination. Boundaries of the internal elastic lamina could always be identified in the histologic sections. Echographically, the internal elastic lamina was not always a distinct bright echo structure. For the determination of plaque thickness the junction plaque 
hypoechoic media was used instead. In the presence of a significant lesion the hypoechoic media became invisible. In these instances the maximal plaque thickness envisioned was determined. In addition, lesions that contained calcium or significant collagen were casting ultrasonic shadowing preventing visualization of the media and adventitia.

\section{RESULTS}

\section{Morphology}

It was found that intravascular ultrasound could distinguish muscular arteries from elastic arteries based on the reflectivity of the media. Elastic arteries (aorta, carotid) exhibit a media densely packed with elastin fibres. In contrast, a muscular artery is composed of smooth muscle cells and hardly any elastin fibres are present.
On ultrasound, a muscular artery presents with a three-layered structure. Internal elastic lamina and adventitia both present as bright echoes circumscribing the hypoechoic media (Figs 2 and 3). In the elastic type of artery no distinction is possible between the various wall components (Fig. 2).

The echo characteristics of the lesions involved were classified as hypoechoic, soft or bright, with or without shadowing. Local deposits of lipid seen in the histological section corresponded to a hypoechoic area in a lesion. Diffuse intimal thickening of a lesion, mainly composed of fibromuscular cells with disorderly arrangement of elastin fibres, produced homogenous soft to bright echoes. When collagen-rich fibrous tissue or calcified deposits were present bright echoes were seen (Figs 2 and 3 ). In the presence of calcification shadowing occurred whereas a large amount of collagen tissue in the lesion prevented identification of the more remote vascular structures.
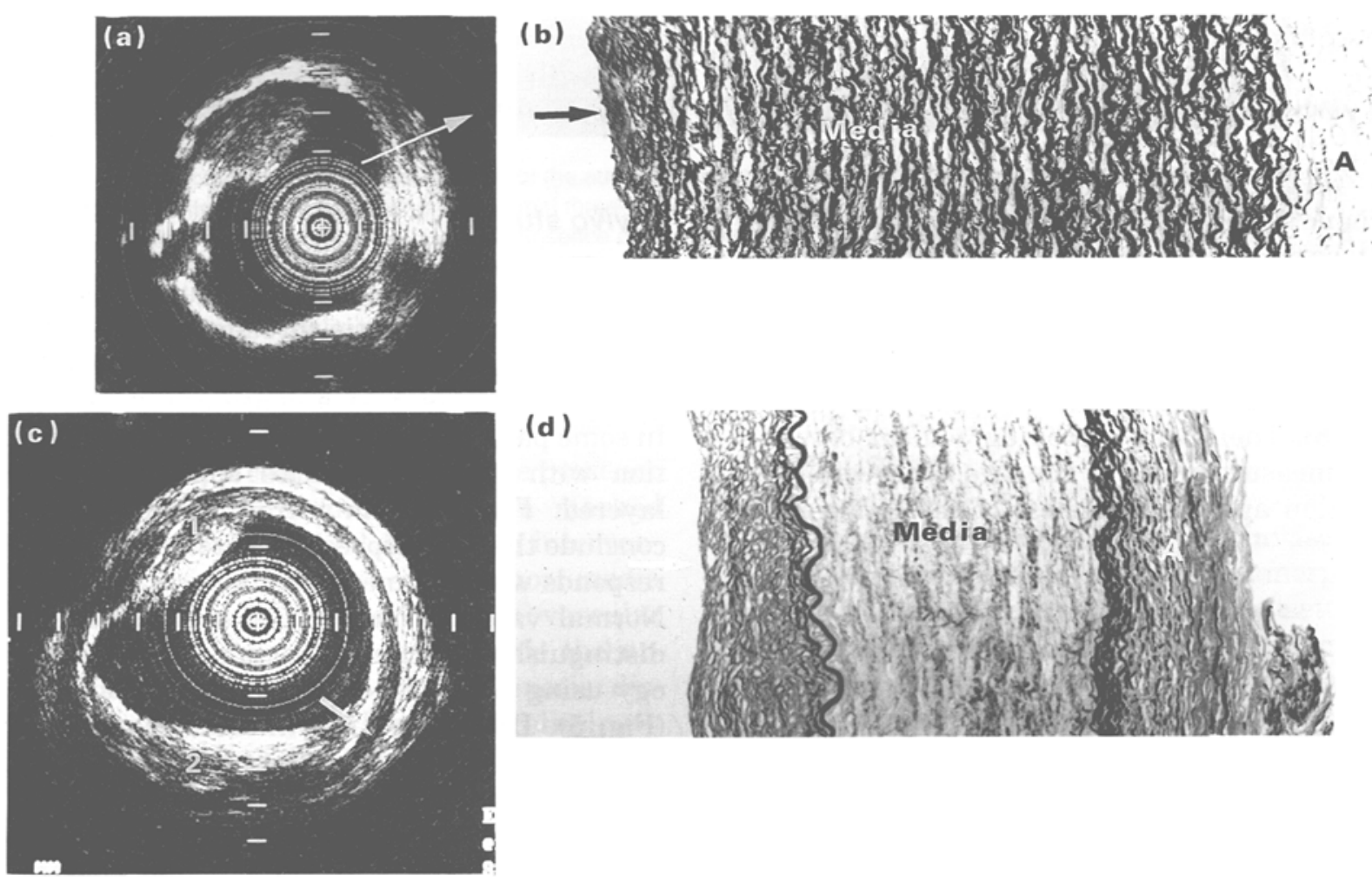

(d)

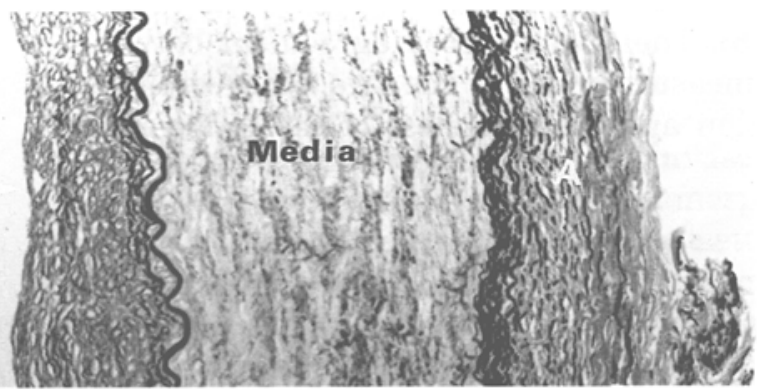

Fig. 2. Ultrasonic and corresponding detail of a histological cross-section showing the characteristics of an elastic, carotid (upper panel) and a muscular femoral artery (lower panel). The media of an elastic artery is densely packed with elastin fibres (detail; elastin fibres stained black) appearing as bright echoes indistinguishable from its counterparts. The media of a muscular artery, mainly composed of smooth muscle cells, appears as a hypoechoic zone interposed between internal elastic lamina and adventitia (A). The carotid artery is severely affected by atherosclerosis. Calcium with shadowing is noted between 6 and 11 o'clock. Superimposed thrombus (at 11 o'clock) is characterized as soft echoes. Detail histology corresponds with $20^{\prime}$ clock (arrow). The femoral artery (lower panel) shows two eccentric lesions ( 1 and 2). The lesion is mainly composed of fibroelastic tissue. Hypoechoic area inside the lesion corresponded with the lipid deposits. Calibration $=1 \mathrm{~mm}$. 

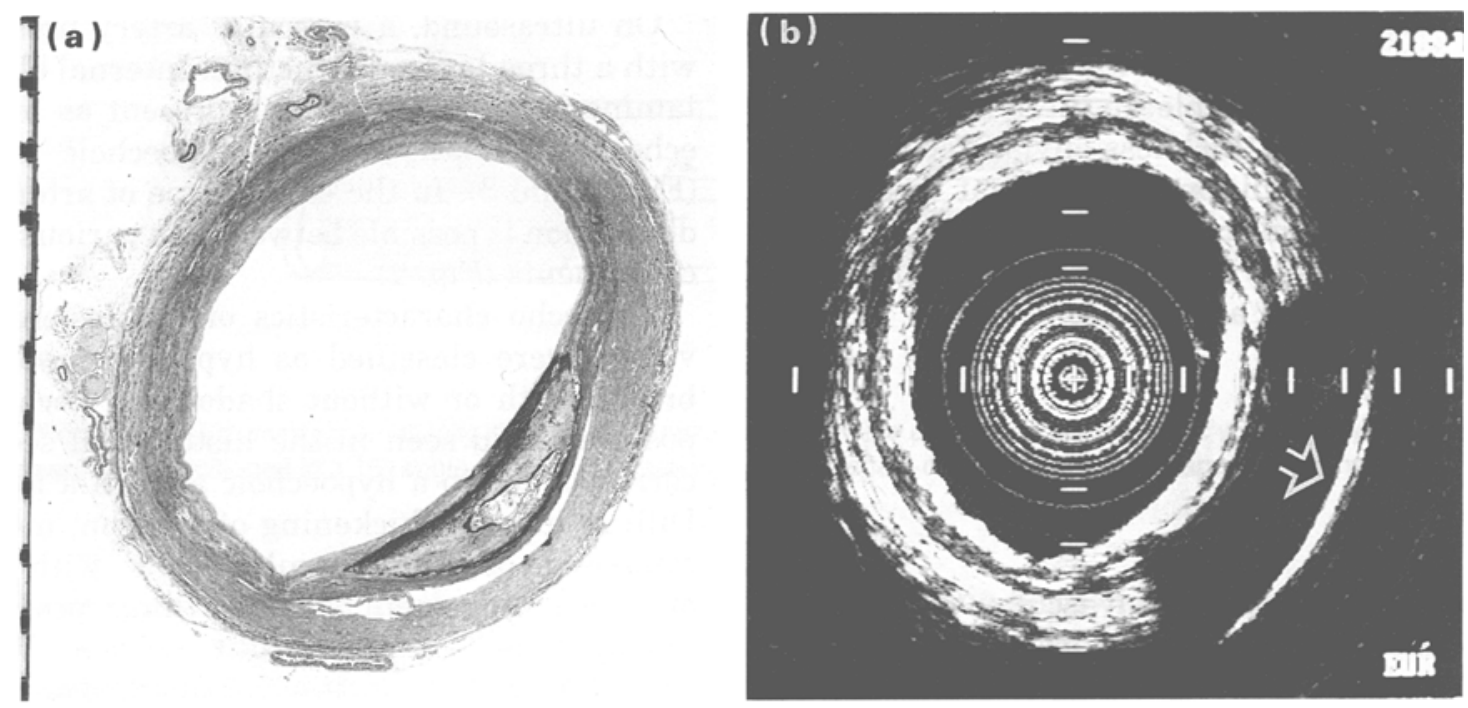

Fig. 3. Microphotograph of a histological section and corresponding intravascular ultrasonic cross-section obtained from a muscular artery (iliac) with advanced atherosclerotic lesion. For the majority of the vessel circumference the lesion was of a fibromuscular and dense fibrous tissue. Between 3 and $6 o^{\prime}$ 'clock a distinct atherosclerotic lesion is seen characterized by bright echoes. Due to attenuation and reflection of the ultrasound caused by calcification in this lesion, no echoes were derived from the media or adventitia. The media of a muscular artery is characteristically hypoechoic. Note the non-structural echo (open arrow) caused by reverberation between the lesion and the transducer. Haematoxylin and azophloxine stain. Calibration $=1 \mathrm{~mm}$.

\section{Quantification}

\section{In vitro studies}

It was generally impossible to document plaque thickness in the elastic arteries as no ultrasonic distinction was noted between the various layers. In a group of 39 specimens in which the media was hypoechoic, plaque thickness could be calculated. Of the lesions present in 36 of 39 arteries, 33 were eccentric (Figs 2 and 3 ). There was a good agreement between the measurements of the two observers. The location and the thickness of the lesion mea-

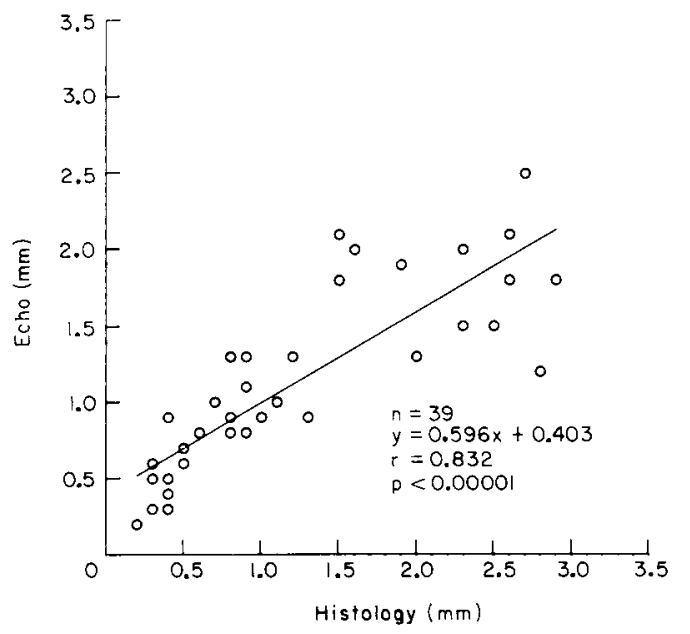

sured from the histological section correlated well with the data derived from the corresponding ultrasound image (Fig. 4).

\section{In vivo studies}

In vivo intravascular ultrasound images showed a characteristic three-layered appearance of the iliac, femoral and popliteal arteries. The aorta was seen as a dense echostructure. In some patients the distal aorta near the junction with the iliac arteries appeared threelayered. From the in vitro information we conclude that the hypoechoic vascular wall corresponds with the media of a muscular artery. Normal vascular morphology could easily be distinguished from cross-sections with pathology using the hypoechoic media as landmark (Fig. 5). Diffuse intimal thickening could be discriminated from the atherosclerotic lesions

Fig. 4. Maximum plaque thickness measured from the histological section and the ultrasound section. Note close relationship in the measurements obtained with both techniques. 

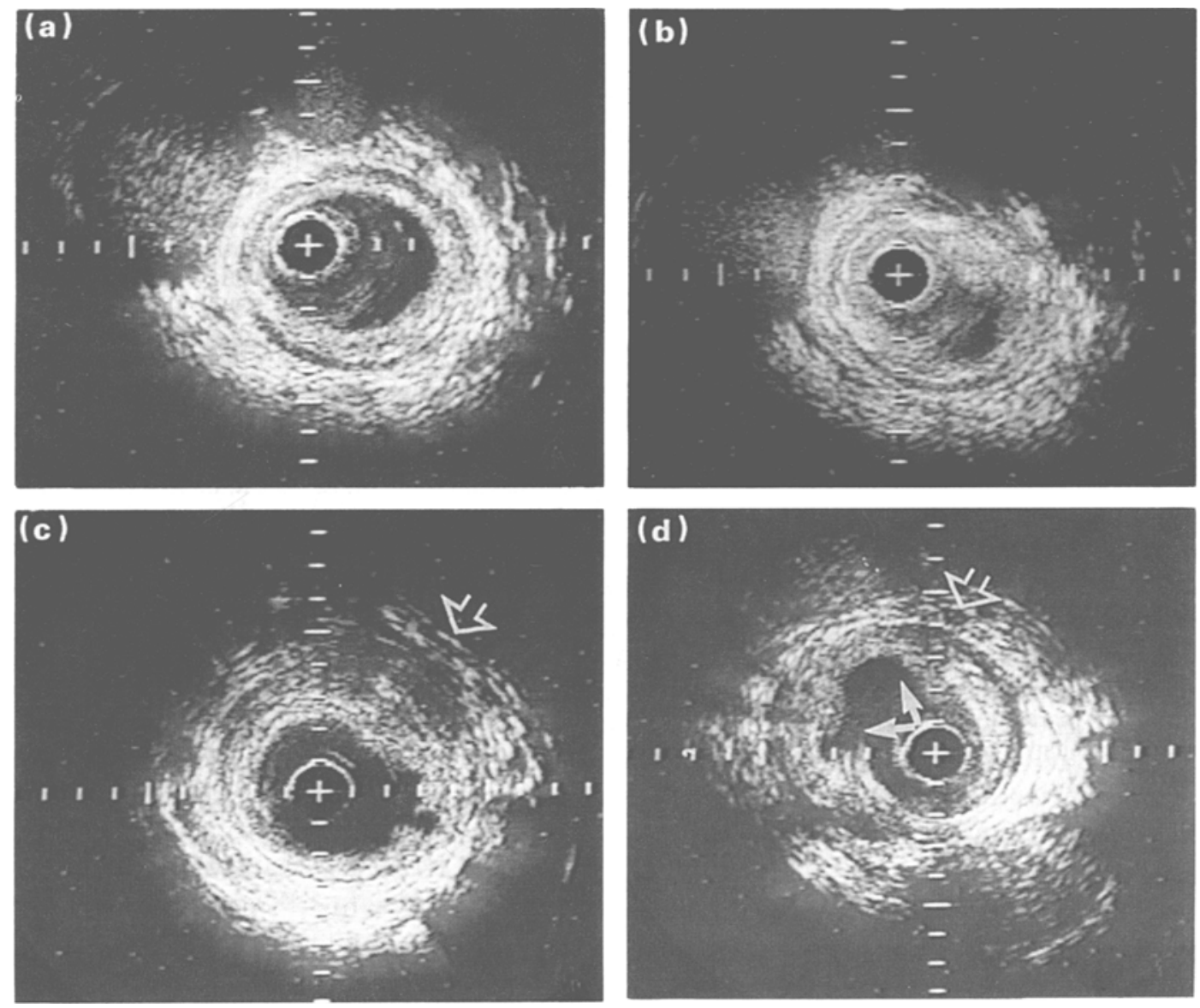

Fig. 5. In vivo obtained arterial cross-sections of the superficial femoral artery $(a, b)$ following balloon angioplasty (c, d) using high frequency intravascular ultrasound. (a) Normal three-layered appearance with diffuse intimal thickening. (b) Significant narrowing is seen with calcium at $10^{\prime}$ clock and absence of the three-layered appearance between 1 and 5 o'clock.

(c, d) Increased lumen area revealing plaque rupture and dissection. (c) The hypoechoic area in the lesion (open arrow) may correspond to an atheroma. In the plaque-free arterial wall, seen between 5 and 9 o'clock, the muscular hypoechoic media is visible and thinned. (d) Cross-section, $3 \mathrm{~cm}$ remote from the previous described section revealing plaque rupture (arrow) and dissection (open arrow). Calibration $=1 \mathrm{~mm}$.

as the latter are characterized by the presence of calcium or collagen resulting in marked attenuation of ultrasound. Following intervention (balloon dilatation) the hypoechoic media was also used to determine the actual vascular wall, the presence of oedema outside the arterial lumen, the location of rest stenosis, dissection or perforation (Fig. 5). Preliminary results indicate that following balloon angioplasty, an increased free lumen area was generally associated with an increase in original lumen area. In this respect it was essential to have an hypoechoic media visible in order to delineate the original lumen area as well as the lesion area. The lesion area, however, in the majority of cross-sections remained practically unchanged.

\section{CONCLUSION}

This study demonstrates that intravascular high frequency ultrasound can detect morphology in both an in vitro and in vivo setting. With further clinical validation intravascular high frequency ultrasound imaging will play an important diagnostic, therapeutic and prognostic role. Diagnosis and characterization of arterial atherosclerotic disease offers interesting research and clinical possibilities, such as staging its severity and the study of its natural history. It opens exciting perspectives for studying regression of atherosclerosis, whether using a cholesterol-lowering diet or drugs. Based on detailed information on the wall pathology and its severity, the interventionist can select the most appropriate therapeutic device for a specific lesion. Intravascular ultra- 
sonic imaging could be of major help during and after mechanical atherectomy and angioplasty as a guidance tool and for deciding the end-point of the intervention.

In conclusion, intravascular real-time high frequency echography is an exciting new development and offering new horizons for clinical research. Practical applications are rapidly emerging. In the near future it may become a major adjunct to second-generation angioplasty procedures, as a guidance tool and for the immediate evaluation of results, especially as it is easier to use and provides unique information much faster than other imaging modalities.

\section{ACKNOWLEDGEMENTS}

This project was supported by grants from the Interuniversity Cardiology Institute of The Netherlands and The Netherlands Technology Foundation (STW) under grant number RGN.77.1257.

\section{REFERENCES}

1 Bom N, ten Hoff H, Lancée CT et al. Early and recent intraluminal ultrasound devices. Int $J$ Cardiac Imaging $1989,4: 79-88$

2 Bom N, Lancée CT, van Egmond FC. An ultrasonic intracardiac scanner. Ultrasonic 1972, 10:72-6.
3 Bom N, Lancée CT, Slager CJ et al. Ein Weg zur Intraluminaren Echoarteriographie. Ultraschall 1987, 8:233-6

4 Bom N, Slager CJ, van Egmond FC et al. Intra-arterial ultrasonic imaging for recanalization by spark erosion. Ultrasound Med Biol 1988, 14:257-61.

5 Hodgson JMcB, Graham SD, Savaku AD et al. Clinical percutaneous imaging of coronary anatomy using an over-the-wire ultrasound catheter system. Int $J$ Cardiac Imaging 1989, 4:187-93

6 Bom N, Hoff H, Lancée CT et al. Early and present examples of intraluminal ultrasonic echography. SPIE 1989, 1068:146-50

7 Yock PG, Johnson EL, Linker DT. Intravascular ultrasound: development and clinical potential. Am J Card Imaging 1988, 2:185-93

8 Gussenhoven WJ, Essed CR, Lancée CT et al. Arterial wall characteristics determined by intravascular imaging: an in vitro study. $J$ Am Coll Cardiol 1989, 14:94752

9 Gussenhoven WJ, Essed CE, Frietman P et al. Intravascular echographic assessment of vessel wall characteristics: a correlation with histology. Int $J$ Cardiac Imaging 1989, 4:105-16

10 Gussenhoven WJ, Essed CE, Frietman P et al. Intravascular ultrasonic imaging: histologic and echographic correlation. Eur J Vasc Surg 1989, 3:571-6

11 Roelandt JR, Bom N, Serruys PW et al. Intravascular high-resolution real-time cross-sectional echocardiography. Echocardiography 1989, 6:1-8

12 Lillie RD, Fullmer HM. Connective tissue fibres and membranes. In: Histopathologic technique and practical histochemistry. London: McGraw-Hill Company, 1976:679-718

Key words: Intravascular ultrasound; Vascular morphology 